\title{
Inertial Effects on Neutrino Oscillations
}

\author{
S. Capozziello* and G. Lambiase ${ }^{\dagger}$ \\ Dipartimento di Scienze Fisiche "E.R. Caianiello" \\ Universitá di Salerno, 84081 Baronissi (Sa), Italy. \\ Istituto Nazionale di Fisica Nucleare, Sez. di Napoli, Italy.
}

July 30, 2018

\begin{abstract}
The inertial effects on neutrino oscillations induced by the acceleration and angular velocity of a reference frame are calculated. Such effects have been analyzed in the framework of the solar and atmospheric neutrino problem.
\end{abstract}

PACS number(s): 14.60.Pq, 95.30.Sf

Keyword(s): Neutrino oscillations, Gravitational field

\footnotetext{
*E-mail: capozziello@physics.unisa.it

${ }^{\dagger}$ E-mail: lambiase@physics.unisa.it
} 


\section{Introduction}

The long-standing problem about the deficiency of solar neutrino, i.e. a discrepancy between the measured $\nu_{e}$ flux predicted by various solar models [1], [2] and the atmospheric neutrino problem [3], might be explained invoking oscillations between the various flavors or generations of neutrinos. It is well known, in fact, that neutrino oscillations 《⿻ can occur, in the vacuum, if the eigenvalues of the mass matrix are not all degenerate, and the corresponding mass eigenstates are different from weak interaction eigenstates $\nu_{e}, \nu_{\mu}, \nu_{\tau}$. The most often discussed version of this type of solutions is the MikheyevSmirnov-Wolfeinstein effect [5] in which the solar electron neutrinos can be converted, almost completely, into muon or tau neutrinos due to the presence of matter in the Sun. Recently a quantum field theory of neutrino oscillations has been proposed by Blasone, Vitiello [6] and Sassaroli [7].

An alternative mechanism of neutrino oscillations, which does not require neutrino to have a non-zero mass, was first suggested by Gasperini [8] and by Halprin and Leung [9] as a means to test the equivalence principle. In this mechanism, neutrino oscillations occur as a consequence of an assumed flavor non-diagonal coupling of neutrinos to gravity which violates the equivalence principle. This line of research has been followed also in Refs. [10]. A new solution of the solar neutrino problem has been proposed in Ref. [11] by using the mechanism introduced by Ellis, Hagelin, Nanopoulos and Srednicki [12], in which the effect of quantum mechanics violation, due to quantum gravity on neutrino oscillation, is investigated.

The effect of gravitationally induced quantum mechanical phases in neutrino oscillation have been discussed in Ref. 13. Ahluwalia and Burgard consider the gravitational effect on the neutrino oscillations showing that an external weak gravitational field of a star adds a new contribution to the phase difference. They also suggest that the new oscillation phase may be a significant effect on the supernova explosions since the extremely large fluxes of neutrinos are produced with different energies corresponding to the flavor states. This result has been critically riexamined by Bhattachya, Habib and Mottola [14]. They showed that the possible gravitational effect appears at higher order with respect to that calculated in Ref. [13], with a magnitude of the order $10^{-9}$, which is completely negligible in typical astrophysical applications.

Neutrino oscillations in curved space time have been also studied by Piniz, Roy, Wudka [15], which observe that spin flavor resonant transitions of neutrinos may occur in vicinity of active galactic nuclei due to gravitational effects and presence of a large magnetic field, and by Cardall and Fuller [16] which introduce an approach to show that gravitational (like Schwarzschild field) effects on neutrino oscillations are intimately related to the redshift.

The purpose of this paper is to calculate the contribution to neutrino oscillations induced by inertial effects arising from acceleration and rotation of reference frames. As it is well known, these effects are relevant in interferometry experiments. In fact, by

using an accelerated neutron interferometer, Bonse and Wroblewski were able to find the 
predict phase shift [17]. Due to the validity of the equivalence principle, one expects that this effect occurs also in a gravitational field, as verified by Colella, Overhausen, Werner [18]. Besides, Mashhoon has derived a coupling of neutron spin to the rotation of a non-inertial reference frame [19] from an extension of the hypothesis of locality, Atwood et al. found the neutron Sagnac effect using an angular velocity of about 30 times that of Earth [20], and finally, Papini, Cai, Lloyd calculate the spin-rotation and spin-acceleration contributions to the helicity precession of fermions [21].

At the present, there is a strong evidence in favour of oscillations of solar and atmospheric neutrinos and of their non-zero masses. Such results have been found in different experiments: 1) solar neutrino experiments [22, 23, 24, 25, 26], 2) atmospheric neutrino experiments [27, 28, 29, 30, 31], and 3) the accelerator LSND experiment [32]. Nevertheless, we have to note that many other neutrino oscillation experiments with neutrinos produced by reactors and accelerators did not find any evidence of neutrino oscillations.

Recent reports indicate that the best fit in favour of neutrino oscillations are obtained for the following cases 33]:

(MSW) small angle mixing region

$$
\left|m_{2}^{2}-m_{1}^{2}\right| \simeq(3 \div 10) \cdot 10^{-6} \mathrm{eV}^{2}, \quad \sin ^{2} 2 \theta \simeq(0.6 \div 1.3) \cdot 10^{-2} ;
$$

(MSW) large angle mixing region

$$
\left|m_{2}^{2}-m_{1}^{2}\right| \simeq(1 \div 20) \cdot 10^{-5} \mathrm{eV}^{2}, \quad \sin ^{2} 2 \theta \simeq 0.5 \div 0.9 ;
$$

solar vacuum oscillation

$$
\left|m_{2}^{2}-m_{1}^{2}\right| \simeq(0.5 \div 1.1) \cdot 10^{-10} \mathrm{eV}^{2}, \quad \sin ^{2} 2 \theta \simeq 0.67 \div 1 ;
$$

atmospheric neutrino oscillation (see also [34, 35])

$$
\begin{gathered}
\left|m_{2}^{2}-m_{1}^{2}\right| \simeq\left(10^{-3} \div 10^{-2}\right) \mathrm{eV}^{2}, \quad \sin ^{2} 2 \theta \geq 0.8 \\
\left|m_{2}^{2}-m_{1}^{2}\right| \simeq(0.5 \div 6) \cdot 10^{-3} \mathrm{eV}^{2}, \quad \sin ^{2} 2 \theta \geq 0.82
\end{gathered}
$$

LSND experiment

$$
\left|m_{2}^{2}-m_{1}^{2}\right| \simeq(0.2 \div 10) \mathrm{eV}^{2}, \quad \sin ^{2} 2 \theta \simeq(0.2 \div 3) \cdot 10^{-2}
$$

$\left|m_{2}^{2}-m_{1}^{2}\right|$ is the mass-squared difference of neutrinos and $\theta$ the mixing angle. In the following, we will restrict to consider only the cases of solar and atmospheric neutrino oscillations in the vacuum.

The layout of the paper is the following. In Sect. 2 we shortly discuss Dirac equation in curved space-time and we calculate the probability that neutrino flavor oscillations occur with respect to an accelerating and rotating observer. In Sect. 3 we discuss the phenomenological consequences of inertial effects on solar and atmospheric neutrino problem. Conclusions are drawn in Sect. 4. 


\section{Neutrino Oscillations Induced by Accelerations and Rotations}

As in Ref. [16], the generalized neutrino phase is given by

$$
\left|\psi_{f}(\lambda)>=\sum_{j} U_{f j} e^{i \int_{\lambda_{0}}^{\lambda} P \cdot p_{n u l l} d \lambda^{\prime}}\right| \nu_{j}>
$$

where $f$ is the flavor index and $j$ the mass one. $U_{f j}$ are the matrix elements transforming flavor and mass bases, $P$ is the four-momentum operator generating space-time translation of the eigenstates and $p_{\text {null }}^{\mu}=d x^{\mu} / d \lambda$ is the tangent vector to the neutrino worldline $x^{\mu}$, parameterized by $\lambda$. The covariant Dirac equation in curved space-time [36] is $\left[i \gamma^{\mu}(x) D_{\mu}-m c / \hbar\right] \psi=0$, where the matrices $\gamma^{\mu}(x)$ are related to the usual Dirac matrices $\gamma^{\hat{a}}$ by means of the vierbein fields $e_{\mu}^{\hat{a}}(x)$, where the Greek (Latin with hat) indices refer to curved (flat) space-time. $D_{\mu}$ is defined as $D_{\mu}=\nabla_{\mu}+\Gamma_{\mu}(x)$, where $\nabla_{\mu}$ is the usual covariant derivative and $\Gamma_{\mu}(x)$ is the spinorial connection defined by

$$
\Gamma_{\mu}(x)=\frac{1}{8}\left[\gamma^{\hat{a}}, \gamma^{\hat{b}}\right] e_{\hat{a}}^{\nu} e_{\nu \hat{b} ; \mu}
$$

(semicolon represents the covariant derivative). The relations

$$
\gamma^{\hat{a}}\left[\gamma^{\hat{b}}, \gamma^{\hat{c}}\right]=2 \eta^{\hat{a} \hat{b}} \gamma^{\hat{c}}-2 \eta^{\hat{a} \hat{c}} \gamma^{\hat{b}}-2 i \varepsilon^{\hat{d} \hat{a} \hat{b} \hat{c}} \gamma^{5} \gamma^{\hat{d}}
$$

where $\eta^{\hat{a} \hat{b}}$ is the metric tensor in flat spacetime, $\varepsilon^{\hat{d} \hat{a} \hat{b} \hat{c}}$ is the totally antisymmetric tensor, $\gamma^{5}=i \gamma^{\hat{0}} \gamma^{\hat{1}} \gamma^{\hat{2}} \gamma^{\hat{3}}$ and $\left\{\gamma^{5}, \gamma^{\hat{a}}\right\}=0$, allow to recast the non-vanishing contribution from the spin connection in the form

$$
\gamma^{\hat{a}} e_{\hat{a}}^{\mu} \Gamma_{\mu}=\gamma^{\hat{a}} e_{\hat{a}}^{\mu}\left\{i A_{G \mu}\left[-(-g)^{-1 / 2} \frac{\gamma^{5}}{2}\right]\right\}
$$

where

$$
A_{G}^{\mu}=\frac{1}{4} \sqrt{-g} e_{\hat{a}}^{\mu} \varepsilon^{\hat{d} \hat{a} \hat{b} \hat{c}}\left(e_{\hat{b} \mu ; \sigma}-e_{\hat{b} \sigma ; \nu}\right) e_{\hat{c}}^{\nu} e_{\hat{d}}^{\sigma},
$$

and $g \equiv \operatorname{det}\left(g_{\mu \nu}\right) . g_{\mu \nu}$ is the metric tensor of curved space-time. The momentum operator $P_{\mu}$, used to calculate the phase of neutrino oscillations, is derived from the mass shell condition

$$
\left(P_{\mu}+\hbar A_{G \mu} \gamma^{5}\right)\left(P^{\mu}+\hbar A_{G}^{\mu} \gamma^{5}\right)=-M_{f}^{2} c^{2},
$$

where $M_{f}^{2}$ is the vacuum mass matrix in flavor base

$$
M_{f}^{2}=U\left(\begin{array}{cc}
m_{1}^{2} & 0 \\
0 & m_{2}^{2}
\end{array}\right) U^{\dagger}, \quad U=\left(\begin{array}{cc}
\cos \theta & \sin \theta \\
-\sin \theta & \cos \theta
\end{array}\right) .
$$


$\theta$ is the vacuum mixing angle. Ignoring terms of the order $\mathcal{O}\left(\hbar^{2} A_{G}^{2}\right)$ and $\mathcal{O}\left(\hbar A_{G} M_{f}\right)$, one gets that, for relativistic neutrinos moving along generic trajectories parameterized by $\lambda$, the column vector of flavor amplitude

$$
\chi(\lambda)=\left(\begin{array}{c}
<\nu_{e} \mid \psi(\lambda)> \\
<\nu_{\mu} \mid \psi(\lambda)>
\end{array}\right)
$$

satisfies the equation

$$
i \frac{d \chi}{d \lambda}=\left(\frac{M_{f}^{2} c^{2}}{2}+\hbar p \cdot A_{G} \gamma^{5}\right) \chi
$$

In deriving Eq. (7), one uses the relation $P^{0}=p^{0}$ and $P^{i} \approx p^{i}$ [16. In an accelerating and rotating frame, the vierbein fields $e_{\mu}^{\hat{a}}(x)$ are given by [37]

$$
e_{0}^{\hat{0}}=1+\frac{\vec{a} \cdot \vec{x}}{c^{2}}, \quad e_{m}^{\hat{0}}=0, \quad e_{0}^{\hat{k}}=\varepsilon^{\hat{k} \hat{l} \hat{m}} \omega^{\hat{l}} x^{\hat{m}}, \quad e_{l}^{\hat{k}}=\delta_{l}^{k},
$$

where $k, l, m=1,2,3, x^{\mu}=\left(x^{0}, \vec{x}\right)$ are the local coordinates for the observer at the origin and $\vec{a}, \vec{\omega}$ are the acceleration and angular velocity of the frame, respectively. The components $e_{\hat{a}}^{\mu}(x)$ and $e_{\mu \hat{a}}(x)$ are calculated by using the metric tensors $g_{\mu \nu}$ and $\eta_{\hat{a} \hat{b}}$, with $g_{\mu \nu}$ given by the element line [37]

$$
d s^{2}=\left[\left(1+\frac{\vec{a} \cdot \vec{x}}{c^{2}}\right)^{2}+\left(\frac{\vec{\omega} \cdot \vec{x}}{c}\right)^{2}-\frac{(\vec{\omega} \cdot \vec{\omega})(\vec{x} \cdot \vec{x})}{c^{2}}\right]\left(d x^{0}\right)^{2}-2 d x^{0} d \vec{x} \cdot \frac{(\omega \wedge \vec{x})}{c}-d \vec{x} \cdot d \vec{x} .
$$

Eq. (9) has been derived by the following consideration (see [37]): a local inertial frame accelerates and rotates relative to Earth (we are considering Earth in view of the following discussion where the detector of neutrinos is comoving with it), owing to earth's gravity and rotation. Then, as a consequence, the local physics in this frame is that of special relativity, provided that the effects induced by the curvature can be neglected. In this meaning, an observer in a "stationary laboratory" on Earth finds himself in a noninertial frame, and inertial effects arise due to the acceleration and rotation.

Inserting Eq. (8) into (3), one gets the components of $A_{G}^{\mu}$

$$
A_{G}^{0}=0, \quad \vec{A}_{G}=\frac{\sqrt{-g}}{2} \frac{1}{1+\frac{\vec{a} \cdot \vec{x}}{c^{2}}}\left\{2 \frac{\vec{\omega}}{c}-\frac{1}{c^{2}}[\vec{a} \wedge(\vec{x} \wedge \vec{\omega})]\right\},
$$

so that Eq. (7) becomes

$$
i \frac{d}{d \lambda}\left(\begin{array}{c}
a_{e} \\
a_{\mu}
\end{array}\right)=\mathcal{T}\left(\begin{array}{l}
a_{e} \\
a_{\mu}
\end{array}\right)
$$

where $a_{f} \equiv<\nu_{f} \mid \psi(\lambda)>, f=e, \mu$ and the matrix $\mathcal{T}$ is defined as

$$
\mathcal{T}=\left[\begin{array}{cc}
-(\Delta / 2) \cos 2 \theta & (\Delta / 2) \sin 2 \theta-\hbar \vec{p} \cdot \vec{A}_{G} \\
(\Delta / 2) \sin 2 \theta-\hbar \vec{p} \cdot \vec{A}_{G} & (\Delta / 2) \cos 2 \theta
\end{array}\right]
$$


up to the $\left(m_{1}^{2}+m_{2}^{2}\right) c^{2} / 2$ term, proportional to identity matrix. Here $\Delta \equiv\left(m_{2}^{2}-m_{1}^{2}\right) c^{2} / 2$. We restrict to flavors $e, \mu$, but this analysis works also for different neutrino flavors. To determine the mass eigenstates $\mid \nu_{1}>$ and $\mid \nu_{2}>$, corresponding to a fixed value of the acceleration and angular velocity of the frame (i.e. for a fixed value of the affine parameter $\lambda$ ), one has to diagonalize the matrix $\mathcal{T}$. Using the standard procedure, one writes the mass eigenstates as a superposition of flavor eigenstates

$$
\begin{aligned}
& \left|\nu_{1}(\lambda)>=\cos \tilde{\theta}(\lambda)\right| \nu_{e}>-\sin \tilde{\theta}(\lambda) \mid \nu_{\mu}>, \\
& \left|\nu_{2}(\lambda)>=\sin \tilde{\theta}(\lambda)\right| \nu_{e}>+\cos \tilde{\theta}(\lambda) \mid \nu_{\nu}>,
\end{aligned}
$$

where the mixing angle $\tilde{\theta}$ is defined in terms of the vacuum mixing angle

$$
\tan 2 \tilde{\theta}=\frac{\Delta \sin 2 \theta-2 \hbar \vec{p} \cdot \vec{A}_{G}}{\Delta \cos 2 \theta} .
$$

We note that $\tilde{\theta} \rightarrow \theta$ as $\vec{A}_{G} \rightarrow 0$ (i.e. $\vec{a} \rightarrow 0, \vec{\omega} \rightarrow 0$ ). The corresponding eigenvalues are

$$
\tau_{1,2}= \pm \sqrt{\frac{\Delta^{2}}{4} \cos ^{2} 2 \theta+\left[\frac{\Delta}{2} \sin 2 \theta-\left(\vec{p} \cdot \vec{A}_{G}\right)\right]^{2}} .
$$

Writing $\left|\psi(\lambda)>=a_{1}(\lambda)\right| \nu_{1}>+a_{2}(\lambda) \mid \nu_{2}>$, Eq. (11) assumes the form

$$
i \frac{d}{d \lambda}\left(\begin{array}{l}
a_{1} \\
a_{2}
\end{array}\right)=\left(\begin{array}{cc}
\tau_{1} & 0 \\
0 & \tau_{2}
\end{array}\right)\left(\begin{array}{l}
a_{1} \\
a_{2}
\end{array}\right)
$$

where $a_{i}=<\nu_{i} \mid \psi(\lambda)>, i=1,2$, and

$$
\left(\begin{array}{l}
a_{1} \\
a_{2}
\end{array}\right)=\tilde{U}\left(\begin{array}{l}
a_{e} \\
a_{\mu}
\end{array}\right), \quad \tilde{U}=\left(\begin{array}{cc}
\cos \tilde{\theta} & \sin \tilde{\theta} \\
-\sin \tilde{\theta} & \cos \tilde{\theta}
\end{array}\right) .
$$

We used the condition $d \tilde{\theta} / d \lambda \approx 0$ in order that (16) is a diagonal matrix. It means that we are neglecting the variations of acceleration and angular velocity, with respect to the affine parameter $\lambda$, in comparing to their magnitudes. Eq. (16) implies $a_{i}(\lambda)=$ $a_{i}(0) \exp \alpha(\lambda), \alpha(\lambda) \equiv i \int_{\lambda_{0}}^{\lambda} \tau_{i} d \lambda^{\prime}, i=1,2$. For the initial condition $\left.|\psi(0)>=| \nu_{e}\right\rangle$, the state $\mid \psi(\lambda)>$ is

$\left|\psi(\lambda)>=\left[\cos \theta_{0} \cos \tilde{\theta} e^{i \alpha}+\sin \theta_{0} \sin \tilde{\theta} e^{-i \alpha}\right]\right| \nu_{e}>+\left[-\cos \theta_{0} \sin \tilde{\theta} e^{i \alpha}+\sin \theta_{0} \cos \tilde{\theta} e^{-i \alpha}\right] \mid \nu_{\mu}>$,

where $\theta_{0}=\tilde{\theta}\left(\lambda_{0}\right)$. The probability to observe an electronic neutrino is therefore

$$
\left|<\nu_{e}\right| \psi(\lambda)>\left.\right|^{2}=\cos ^{2}\left(\theta_{0}+\tilde{\theta}\right) \sin ^{2} \alpha+\cos ^{2}\left(\theta_{0}-\tilde{\theta}\right) \cos ^{2} \alpha
$$

Eq. (19) shows that accelerating and rotating observers will experience a flavor oscillation of neutrinos. Due to the equivalence principle, one concludes that gravitational fields can induce neutrino oscillations, in agreement with Refs. [8-16]. It is interesting to discuss some particular case, i.e. the frame is accelerating or rotating, in order to estimate the contributions to neutrino oscillations when inertial effects are taken into account. 


\section{Inertial Effects on Solar and Atmospheric Neutri- nos}

Consequences on neutrino oscillations can be derived from Eqs. (12) and (14). Let us suppose that the linear acceleration is zero, $\vec{a}=0$, and the reference frame is rotating. In this situation, one can define a critical angular velocity $\omega_{c}$ such that the off-diagonal matrix elements of (12) vanish

$$
\Delta \sin 2 \theta \approx \frac{2 \hbar}{c} \vec{\omega}_{c} \cdot \vec{p}
$$

implying that $\tilde{\theta}$ can assume the values $\tilde{\theta} \approx 0$ and $\tilde{\theta} \approx \pi / 2$. The consequences of these values are discussed below and only experimental tests may distinguish between them. For ultrarelativistic neutrinos, $E_{\nu} \sim p c$, it reduces to

$$
\left|m_{2}^{2}-m_{1}^{2}\right| \approx \frac{4 \hbar E_{\nu} \omega_{c}}{\sin 2 \theta}
$$

This formula connects the mass-squared difference of neutrinos to the vacuum mixing angle, the neutrino energy and the (critical) angular velocity of the reference frame. In order to infer some consequences from (21) for solar neutrino problem (vacuum oscillations), we assume the reference frame co-moving with the Earth, i.e. its angular velocity is $\omega_{c} \sim 7 \cdot 10^{-5} \mathrm{rad} / \mathrm{sec}$. Results for typical values of the neutrino energies and vacuum mixing angle are reported in Table I. The agreement with the experimental data comes from neutrinos with energy varying in the range $10 \div 60 \mathrm{MeV}$. In this range, we find a mass-squared difference of the order $10^{-12} \div 10^{-10} \mathrm{eV}^{2}$ for vacuum mixing angle $10^{-1} \leq \sin 2 \theta \leq 1$. We have also considered the lower limit $10^{-1}$ for including, in such a way, the actual uncertainty on the values of the vacuum mixing angle and show that, in any case, our results are in very good agreement for $\sin 2 \theta$ belonging to the range $0.1 \div 1$. Besides, we observe that an extreme value of $\tilde{\theta}$ as function of $\theta$ (Eq. (14)) is

$$
\tilde{\theta}=\theta+\frac{\pi}{4}
$$

On the other hand, the condition (20) implies $\tilde{\theta} \approx 0$ or $\tilde{\theta} \approx \pi / 2$, which fix the vacuum mixing angle approximatively to $\theta \approx \pi / 4$, as expected by experimental results for solar neutrinos.

The value $\tilde{\theta} \approx 0$ implies that, for a rotating observer, no mixing occurs since in the flavor oscillations, due to the non-zero mass of neutrinos and to the vacuum mixing angle, the term $\Delta \sin 2 \theta$ is compensated by the geometric term $p \cdot A_{G}$. As a consequence, the neutrino flux is different by the expected one if inertial effects are neglected.

The value $\tilde{\theta} \approx \pi / 2$ induces a conversion phenomena due to which the flux of $\nu_{e}$ component decreases. To be more specific, after the production of electronic neutrinos in the Sun, we have $|\psi(0)>=| \nu_{e}>\left(\theta_{0}=0\right)$. Evolving along its worldline, the $\nu_{e}$ 
component will oscillate in agreement to Eqs. (13). Nevertheless, if condition (14) holds and $\tilde{\theta} \approx \pi / 2$, the probability (19) to find $\nu_{e}$ in the beam decreases from 1 to $\sin ^{2} \theta_{0} \approx 0$. This result shows that the $\nu_{e}$ component of the beam is almost totally depleted with respect to the rotating observer with angular velocity $\omega_{c}$, resulting in a reduction of solar neutrino flux.

Concerning the atmospheric neutrino oscillation, an appreciable value of $\left|m_{2}^{2}-m_{1}^{2}\right|$ requires highly (multiGeV) energetic neutrinos. In fact, Eq. (21) implies $\left|m_{2}^{2}-m_{1}^{2}\right| \sim$ $10^{-4} \mathrm{eV}^{2}$, for $E_{\nu} \sim 10^{4} \mathrm{GeV}$ and $\sin 2 \theta \sim 10^{-2}$. This value of the mixing angle is excluded (at least till now) by experimental data. Using $\sin ^{2} 2 \theta \geq 0.82$, according to the experimental results, Eq. (21) leads to a mass-squared difference of the order $10^{-6} \mathrm{eV}^{2}$, which does not fit the experimental range $10^{-4} \div 10^{-3} \mathrm{eV}^{2}$.

In the regime in which neutrinos are highly energetic so that the condition $\vec{p} \cdot \vec{A}_{G} \gg$ $\Delta \sin 2 \theta$ holds, Eq. (14) implies $\tilde{\theta} \approx \pi / 4$, and the probability to find $\nu_{e}$ component in the neutrino beam is $\approx 1 / 2$, assuming as initial condition $|\psi(0)\rangle=\left|\nu_{e}\right\rangle, \theta_{0}=0$. Highly energetic neutrinos, with energy of the order $1 \div 10^{3} \mathrm{TeV}$, can be produced, for example, by a Supernova [15].

It is interesting to compare the contribution to neutrino oscillations due to the rotation term with that one due to the massive term. Being $p \cdot A_{G}=-(\sqrt{-g} / c) \vec{\omega} \cdot \vec{p}$, Eq. (可) implies that, for ultrarelativistic neutrinos, the angular velocity of the rotating frame is given by

$$
\omega \sim \frac{1}{2} \frac{m_{\nu}^{2} c^{4}}{\hbar E_{\nu}}
$$

If we consider neutrinos with energy $E_{\nu} \sim 1 \mathrm{TeV}$, emanating from active galactic nuclei 15 which are possible sources of high energy signal, being the most luminous objects in the Universe [38], and for $m_{\nu} \sim 1 \mathrm{eV} / c^{2}$ [15], [40], the angular velocity is of the order $\omega \sim 10^{2} \div 10^{3} \mathrm{rad} / \mathrm{sec}$. Moreover, one aspects that the neutrino mass is of the order $m_{\nu} \sim 10^{-2} \div 10^{-4} \mathrm{eV} / c^{2}$ [41, 42]. At energy $E_{\nu} \sim 10 \div 10^{2} \mathrm{GeV}$, produced by accelerators [39], one gets $\omega \sim 1.2 \div 10^{-5} \mathrm{rad} / \mathrm{sec}$.

Some values of the angular velocity, calculated by using Eq. (23) for different (and expected) values of neutrino masses and energies, are reported in Table II. It turns out that these values are of the same order of magnitude of typical angular velocity of astrophysical objects (Table III). For example, the angular velocity $\omega \sim 10^{2} \div 10^{3} \mathrm{rad} / \mathrm{sec}$ is comparable with that one of pulsars [43]. Besides, $\omega \sim 10^{-5} \mathrm{rad} / \mathrm{sec}$ is of the same order of angular velocity of the Earth, and it is about 10 times the angular velocity of the Sun, $\omega_{\text {Sun }} \sim 10^{-6} \mathrm{rad} / \mathrm{sec}$. An experiment (as for example, an interferometer experiment) aimed to measure the quantum mechanical phase shift induced by such a geometric term is difficult to realize since the detector must be comoving with the astrophysical objects. A sensible effect could be tested for Earth assuming the above values of $m \sim 10^{-2} \div 10^{-4} \mathrm{eV} / \mathrm{c}^{2}$ and $E \sim 10 \div 10^{2} \mathrm{GeV}$, but it requires a so high precision that, at the moment, it is not allowed by technology.

In the case in which the acceleration $\vec{a}$ is constant and $\vec{\omega}=0$, the $p \cdot A_{G}$ term in Eq. (匹) vanishes and one finds a shift of the phase: $\Omega=i \int_{\lambda_{0}}^{\lambda} P \cdot p_{\text {null }} d \lambda^{\prime}$, as defined in Eq. (1). 
In fact, since the neutrino trajectory is null and $g_{\mu \nu}$ is diagonal $\left(\sqrt{-g}=1+(\vec{a} \cdot \vec{x}) / c^{2}\right)$, the physical distance can be written as

$$
d \lambda=d l\left(g_{i j} \frac{d x^{i}}{d \lambda} \frac{d x^{j}}{d \lambda}\right)^{-1 / 2}=d l\left[-g_{00}\left(\frac{d x^{0}}{d \lambda}\right)^{2}\right]^{-1 / 2}
$$

and one gets [16]

$$
\Omega=-\frac{M^{2}}{2 E_{*}} \int_{l_{0}}^{l} \frac{1}{1+(\vec{a} \cdot \vec{x}) / c^{2}} d l^{\prime},
$$

where $E_{*}=P_{t}$ is the conserved quantity due to the non dependence of the metric tensor on the timelike coordinates. If $\vec{a} \| \vec{x}, d l=d r$ and Eq. (25) reduces to

$$
\Omega=-\frac{M^{2} c^{2}}{2 E_{*}|\vec{a}|} \ln \left(1+|\vec{a}| r / c^{2}\right)
$$

for $r_{0} \equiv r\left(l_{0}=0\right)=0$. If $\vec{a} \perp \vec{x}$, Eq. (25) gives the standard result $\Omega=-\left(M^{2} c^{2} /\left(2 E_{*}\right)(\lambda-\right.$ $\left.\lambda_{0}\right)$.

\section{Conclusions}

We have analyzed the phenomenological aspects of neutrino oscillations for an accelerating and rotating observer.

The inertial effects on neutrino oscillations seem to be appreciable on solar neutrino problem. The coupling of the angular velocity and momentum of the neutrino implies a reduction of neutrino flux, as experimented by an observer comoving with the Earth, providing us with valuable informations on mass-squared difference and mixing angle of neutrinos. In fact, we find a good agreement between the experimental data [34] and our estimations of the mass-squared differences. They well fit the experimental data for neutrino beams with energies of the order $10 \div 60 \mathrm{MeV}$. For these estimations we have used $\sin 2 \theta \sim 1 \div 10^{-1}$, values coming from the data of solar neutrino oscillation experiments.

In the framework of atmospheric neutrinos, inertial effects seems to be negligible. The best-fit of experimental data that we reproduce, if adiabatic condition holds, comes for highly energetic neutrinos. We get in fact, a value of the mass-squared difference of the order $10^{-4} \mathrm{eV}^{2}$, requiring a mixing angle $\sin 2 \theta \sim 10^{-2}$.

However, we have to note that values of the mass-squared difference of neutrinos and their mixing angle are till now open issues. Only future neutrino oscillation experiments will make possible to investigate in detail the region $\left|m_{2}^{2}-m_{1}^{2}\right|$ and to fix the value of the mixing angle. These data will allow to solve definitely the solar and atmospheric neutrino problems and to understand if inertial effects are important for explaining the deficit of solar and atmospheric neutrino flux. 


\section{Acknowledgment}

The authors would like to thank the referee for the useful comments which allowed to improved the paper.

\section{References}

[1] J.N. Bachall, P.I. Krastev, A. Yu Smirnov, hep-ph/9807216 and reference therein.

[2] J.N. Bachall, M.M. Pinsonneault, Rev. Mod. Phys. 64 (1992) 88; J. Bachall and R.K. Ulrich, Rev. Mod. Phys. 60 (1989) 297; S. Turck-Chieze and I. Lopez, Astr. J. 408 (1993) 347; S. Turck-Chieze et al. Phys. Rep. 230 81993) 57.

[3] K.S. Kirata et al., Phys. Lett. B205 (1988) 416, B280 (1992) 146; R. Becker-Szendy et al., Phys. Rev. D46 (1992) 3720; D. Casper et al., Phys. Rev. Lett. (1991) 2561; W.W.M. Allison et al., Phys. Lett. B391 (1997) 491.

[4] S.M. Bilenky and B. Pontecorvo, Physics Report 41 (1978) 225.

[5] L. Wolfenstain, Phys. Rev. D17 (1978) 2369, Phys. Rev. D20 (1979) 2634; S.P. Mikhyev and A.Yu Smirnov, Yad. Fiz. 42 (1985) 1441 [Sov. J. Nucl. Phys. 42 (1985) 913], Nuovo Cimento C9 (1986) 17.

[6] M. Blasone and G. Vitiello, Ann. Phys. 244 (283) 1995; E. Alfinito, M. Blasone, A. Iorio, G. Vitiello, Phys. Lett. B362 (1995) 91.

[7] E. Sassaroli, Flavor Oscillaton in Field Theory, hep-ph/9609476.

[8] M. Gasperini, Phys. Rev. D38 (1988) 2635, Phys. Rev. D39 (1989) 3606.

[9] A. Halprin and C.N. Leung, Phys. Rev. Lett. 67 (1991) 1833, Nucl. Phys. B (Proc. Suppl.) 28A (1992) 139.

[10] J. Pantaleone, A. Halprin, C.N. Leung, Phys. Rev. D47 (1993) R4199; K. Iida, H. Minakata, O. Yasuda, Mod. Phys. Lett. A8 (1993) 1037; M.N. Bultler, S. Nozawa, R. Malaney, A.I. Boothroyd, Phys. Rev. D47 (1993) 2615.

[11] Y. Liu, L. Hu, M.-L. Ge, Phys. Rev. D56 (1997) 6648.

[12] J. Ellis, J.S. Hagelin, D.V. Nanopoulos, M. Srednicki, Nucl. Phys. B241 (1984) 381.

[13] D.V. Ahluwalia, C. Burgard, Gen. Rel. Grav. 28 (1996) 1161; gr-qc/9606031.

[14] T. Bhattachorya, S. Habib, E. Mottola, Phys. Rev. D59 (1999) 067301.

[15] D. Piniz, M. Roy, J. Wudka, Phys. Rev. D54 (1996) 1587; Phys. Rev. D56 (1997) 2403. 
[16] C.Y. Cardall, G.M. Fuller, Phys. Rev. D55 (1997) 7960.

[17] U. Bonse, T. Wroblewski, Phys. Rev. Lett. 51 (1983) 1401.

[18] R. Colella, A.W. Overhausen, S.A. Werner, Phys. Rev. Lett. 34 (1975) 1472.

[19] B. Mashhoon, Phys. Rev. Lett. 61 (1988) 2639.

[20] D.K. Atwood, M.A. Horne, C.G. Shull, J. Arthur, Phys. Rev. Lett. 52 (1984) 1673.

[21] Y.Q. Cai, D.G. Lloyd, and G. Papini, Phys. Lett. A178 (1993) 225.

[22] B.T. Cleveland et al., Astrophys. J. 496 (1998) 505.

[23] K.S. Hirata et al., Kamiokande Coll., Phys. Rev. Lett. 77 (1996) 1683.

[24] W. Hampel et al., GALLEX Coll., Phys. Lett. B388 (1996) 384.

[25] D.N. Abdurashitov et al., SAGE Coll., Phys. Rev. Lett. 77 (1996) 4708.

[26] Y. Fukuda et al., Super-Kamiokande Coll., Phys. Rev. Lett. 81 (1998) 1158.

Y. Suzuki, Neutrino 98, Takayama, Japan, 1998.

[27] Y. Fuguda et al., Super-Kamiokande Coll., Phys. Rev. Lett. 81 (1998) 1562.

[28] Y. Fuguda et al., Kamiokande Col., Phys. Lett. B335 (1994) 237.

[29] R. Becker-Szendy et al., IMB Coll., Nucl. Phys. B (Proc. Suppl.) 38 (1995) 331.

[30] W.W.M. Allison et al., Soudan-2 Coll., Phys. Lett. B391 (1997) 491.

[31] M. Ambrosio et al., MACRO Coll., preprint hep-ex/9807005 (1998).

[32] C. Athanassopoulos et al., LSND Coll., Phys. Rev. Lett. 81 (1998) 1774.

[33] J.N. Bachall, M.H. Pinsonneault, S. Basu, and J. Christensen-Dolsgaard, Phys. Rev. Lett. 78 (1997) 171.

[34] S.M. Bilenky, C. Giunti, and W. Grimus, hep-ph/9812360 1998.

[35] M.C. Gonzales et al., Phys. Rev. D58 (1998) 033004.

[36] S.W. Weinberg, Gravitation and Cosmology, Wiley, New York, 1972.

N.D. Birrel and P.C.W. Davies, Quantum Fields in Curved Space, Cambridge University Press, Cambridge 1982.

[37] W. Hehl, W.-T. Ni, Phys. Rev. D42 (1990) 2045. 
[38] V.S. Berezin, in Neutrino 77, Proceedings of the International Conference, Bakson Valley, USSR (Nauka, Moscow, 1977), vol. 1, p. 177; D. Eichler, Astrophys. J. 232 (1979) 106; R. Silberberg and M.M Shapiro, in Proceedings of the 16th International Cosmic Ray Conference, Kyoto, Japan 1979, vol. 10, p. 357.

[39] N.J. Bekeret et al., Phys. Rev. Lett. 47 (1981) 1577; N. Ushida et al., ibid. 57 (1986) 2897; L.A. Ahrens et al., Phys. Rev. D31 (1985) 2732.

[40] G.G. Raffaelt, hep-ph/9712292; L.M. Krauss, P.Romanelli, D. Schramm, Nucl. Phys. B380 (1992) 507; D. Cline et al., Phys. Rev. D50 (1994) 720; S.T. Petcov, A.Yu Smirnov, Phys. Lett. B322 (1994) 109; H. Fritzsch, Zhi-Zhong Xing, Phys. Lett. B372 (1996) 265; A. Ioanissyan, J.W.F. Valle, Phys. Lett. B322 (1994) 93; R.N. Mohapatra, S. Nussinov, Phys. Lett. B346 (1995) 75; D.G. Lee, R.N. Mohapatra, Phys. Lett. B329 (1994) 463.

[41] F. Wilczek, hep-ph/9809509; G. Altarelli, hep-th/9809532.

[42] W.C. Haxton, Phys. Rev. D35 (1987) 2352.

[43] M. Harwit, Astrophysical Concepts, Springer-Verlag, New York Inc., 1988. 
Table I: Estimation of $\left|m_{2}^{2}-m_{1}^{2}\right|$ as function of $E_{\nu}, \sin 2 \theta$ and fixed value of $\omega_{c}$.

\begin{tabular}{|c|c|c|}
\hline \hline$E_{\nu}(\mathrm{MeV})$ & $\sin 2 \theta$ & $\left|m_{2}^{2}-m_{1}^{2}\right|\left(\mathrm{eV}^{2}\right)$ \\
\hline \hline 1 & 1 & $10^{-13}$ \\
1 & $10^{-1}$ & $10^{-12}$ \\
\hline \hline 10 & 1 & $10^{-12}$ \\
10 & $10^{-1}$ & $10^{-11}$ \\
\hline \hline $50 \div 60$ & 1 & $10^{-10}$ \\
\hline \hline
\end{tabular}

Table II: Angular velocity of reference frames for different values of neutrino masses and energies.

\begin{tabular}{|c|c|c|}
\hline \hline$m_{\nu}\left(\mathrm{eV} / \mathrm{c}^{2}\right)$ & $E_{\nu}(\mathrm{GeV})$ & $\omega(\mathrm{rad} / \mathrm{sec})$ \\
\hline \hline 1 & $10^{3} \div 10^{-1}$ & $10^{2} \div 10^{6}$ \\
\hline $10^{-2}$ & $10^{3} \div 10^{-1}$ & $10^{-2} \div 10^{2}$ \\
\hline $10^{-4}$ & $10^{3} \div 10^{-1}$ & $10^{-6} \div 10^{-2}$ \\
\hline \hline
\end{tabular}

Table III: Typical angular velocity of Astrophysical objects.

\begin{tabular}{|c|c|}
\hline \hline Astrophysical Objects & $\omega(\mathrm{rad} / \mathrm{sec})[43]$ \\
\hline \hline Pulsar & $4 \cdot 10^{3}$ \\
Sun & $10^{-6}$ \\
Earth & $10^{-5}$ \\
White dwarf & 2.1 \\
RRLyrae Star & $10^{-5}$ \\
Cepheid Variable & $10^{-7}$ \\
\hline \hline
\end{tabular}

